\title{
Perspectivas comunitarias
} alrededor de la desnutrición infantil en tres comunidades wayúus de La Guajira (Colombia)

\author{
Community Perspectives around Child \\ Malnutrition in Three Wayuu Communities \\ in La Guajira (Colombia)
}

Perspectivas da comunidade sobre a desnutrição infantil em três comunidades wayuus de La Guajira (Colômbia)

Jennifer Marcela López-Ríos, MSc ${ }^{1 *}$

Sergio Cristancho, $\mathrm{PhD}^{1}$

Isabel Cristina Posada-Zapata, PhD $^{1}$

Recibido: 30 de julio de 2020 • Aceptado: 2 de febrero de 2021

Doi: https://doi.org/10.12804/revistas.urosario.edu.co/revsalud/a.10286

Para citar este artículo: López-Ríos JM, Cristancho S, Posada-Zapata IC. Perspectivas comunitarias alrededor de la desnutrición infantil en tres comunidades wayúus de La Guajira (Colombia). Rev Cienc Salud. 2021;19(2):1-22.

https://doi.org/10.12804/revistas.urosario.edu.co/revsalud/a.10286

\section{Resumen}

Introducción: la desnutrición infantil es un problema de salud pública que afecta la supervivencia de algunas comunidades indígenas. Varios estudios la han abordado desde la visión biomédica, pero son pocos desde la mirada de dichas comunidades. El objetivo de este artículo es comprender las perspectivas comunitarias relacionadas con la desnutrición en niños de 0 a 7 años de edad en las comunidades wayúus de Taiguaicat, Pañarrer y Limunaka del resguardo Manaure (La Guajira, Colombia). Desarrollo: se realizó un estudio cualitativo como parte de una investigación participativa basada en la comunidad. Se llevaron a cabo 21 entrevistas semiestructuradas, 3 foros comunitarios y un ejercicio de fotovoz con 12 participantes. Se analizó la información con herramientas analíticas de la teoría fundamentada. Los

1 Universidad de Antioquia, Facultad Nacional de Salud Pública, Grupo de Investigación en Salud Mental, Medellín, Antioquia (Colombia). 
hallazgos alrededor de las perspectivas comunitarias de la desnutrición infantil se agruparon en dos categorías analíticas: a) las prácticas arijunas (o las realizadas por los que no son indígenas), de la pérdida de lo ancestral a la enfermedad del hambre, y b) la enfermedad del hambre: su relación con la alimentación del binomio madre-hijo, durante la gestación y después de esta. Conclusiones: la desnutrición infantil en los wayúus es un fenómeno complejo e histórico, con un trasfondo de desigualdades sociales y de estructuras de poder injustas. De acuerdo con los participantes, la supervivencia de la niñez wayúu está siendo amenazada por la desnutrición, la cual afecta principalmente al binomio madre-hijo, ocasionada por el poco acceso y disponibilidad a los alimentos, por las inadecuadas prácticas occidentales en el territorio y por la pérdida de las prácticas tradicionales como la siembra y el pastoreo.

Palabras clave: desnutrición infantil; población indígena; seguridad alimentaria; investigación participativa basada en la comunidad; Colombia.

\section{Abstract}

Introduction: Child malnutrition is a public health problem that has affected the survival of some indigenous communities. Several studies have approached it from the biomedical perspective, although only a few have analyzed it from these communities' perspective. The objective of the present article is to understand the community perspectives related to malnutrition in children aged 0-7 years in the Wayuu communities of Taiguaicat, Pañarrer, and Limunaka del Resguardo Manaure, La Guajira, Colombia. Development: A qualitative study was performed. Additionally, as a part of a participatory community-based research, 21 semi-structured interviews, 3 community forums, and a photovoice exercise were performed with 12 participants. The information was analyzed with analytical tools of the grounded theory. The findings around the community perspectives of child malnutrition were grouped into two analytical categories: (a) the Arijuna practices (or those carried out by non-indigenous people), from the loss of the ancestral to the disease of hunger, and (b) the disease of hunger and its relationship with the feeding of the mother-child binomial during and after pregnancy. Conclusions: Child malnutrition in the Wayuu is a complex historical phenomenon, with a background of social inequalities and unjust power structures. According to the participants' responses, the survival of Wayuu children is threatened by malnutrition, which mainly affects the mother-child relationship caused by poor access to food and its limited availability, inadequate western practices in the territory, and the loss of traditional practices, such as planting and grazing.

Keywords: Child malnutrition; indigenous population; food security; community-based participatory research; Colombia.

\section{Resumo}

Introdução: a desnutrição infantil é um problema de saúde pública que afeta a sobrevivência de algumas comunidades indígenas. Vários estudos têm abordado o tema a partir da perspectiva biomédica, mas poucos são os estudos baseados na perspectiva dessas comunidades. O objetivo deste artigo é compreender as perspectivas da comunidade em relação à desnutrição em crianças de 0 a 7 anos nas comunidades Wayuu de Taiguaicat, Pañarrer e Limunaka del Resguardo Manaure, em La Guajira, na Colômbia. Desenvolvimento: foi realizado um estudo qualitativo no âmbito de uma pesquisa participativa de base comunitária, foram realizadas 21 entrevistas semiestruturadas, 3 fóruns comunitários e um exercício de fotovoz com 12 participantes. As informações foram analisadas com ferramentas analíticas da Teoria Fundamentada. As descobertas em torno das perspectivas da comunidade sobre a desnutrição infantil foram agrupadas em duas categorias analíticas: a) as práticas arijunas, da perda do ancestral à doença da fome, e b) a doença da fome: sua relação com a alimentação do binômio mãe-filho, durante e após a gestação. Conclusões: a desnutrição infantil nos wayuu é um fenômeno complexo e histórico, com um pano de fundo de desigualdades sociais e estruturas de poder injustas. Segundo os participantes, a sobrevivência das crianças wayuu está sendo ameaçada pela desnutrição, que atinge principalmente o binômio mãe-filho, causada pelo pouco acesso e 
disponibilidade de alimentos, pelas práticas ocidentais inadequadas no território e pela perda de práticas tradicionais como a semeadura e o pastejo.

Palavras-chave: desnutrição infantil; população indígena; segurança alimentar; pesquisa participativa de base comunitária; Colômbia.

\section{Introducción}

\section{L}

a desnutrición infantil es un problema de inseguridad alimentaria y de salud pública a descala mundial que se encuentra situado no solamente en un contexto natural y biológico de la enfermedad, sino que es un proceso que involucra dimensiones históricas, sociales, políticas, económicas y culturales. Aflige principalmente a las poblaciones más vulneradas, entre ellas las comunidades indígenas $(1,2)$, de las cuales los niños menores de 5 años son los más afectados por esta situación (2,3), dado que se ha convertido en la principal amenaza para su supervivencia y su crecimiento $(4,5)$. Desde una perspectiva más amplia, la desnutrición infantil se comprende no solo como el resultado de la ingesta insuficiente de alimentos; es también el resultado de otra serie de causas que no son visibles inmediatamente. Algunas de ellas son las subyacentes, como la falta de acceso a los alimentos, a la atención sanitaria y a la adecuada infraestructura de saneamiento básico. También están las básicas o estructurales, como la pobreza, la desigualdad y la exclusión social $(4,6)$.

Colombia no es ajena a este panorama, y la población indígena sigue siendo, hasta hoy, la más desfavorecida, vulnerada y excluida económica, social y políticamente, si se compara con la población no indígena (5,7). De acuerdo con la Encuesta Nacional de Situación Nutricional (ENSIN) 2015, los niños indígenas menores de 5 años presentaron una prevalencia de desnutrición crónica del 29.6\% y una prevalencia de desnutrición global del 7.2\%, una diferencia de más del doble si se compara con la obtenida por la población no indígena, que fue del $10 \%$ y del $3 \%$, respectivamente (8).

En el Departamento de La Guajira, cerca del $45 \%$ de su población es indígena, sobre todo de la etnia wayúu, pueblo conformado por aproximadamente 271000 personas, ubicadas al norte de Colombia, en un territorio donde el clima varía desde lo árido a lo seco, conformado por bosques secos y grandes llanuras desérticas (9). Los wayúus están distribuidos en 21 resguardos, de los cuales los más grandes son los de la Media y la Alta Guajira. Su lengua nativa es el wayuunaiki. Su economía se basa principalmente en el pastoreo, la siembra, el trueque y la artesanía; sin embargo, debido a la actual falta de lluvia en el territorio, no dependen de la agricultura, sino de la comercialización de sus artesanías. Su organización social es por clanes y de carácter matrilineal. Las principales problemáticas que enfrentan como pueblo es la falta de acceso al agua potable, la presencia de grupos armados en su territorio. También "la exploración y explotación de recursos naturales por la presencia de 
multinacionales en el territorio indígena que ha generado un gran daño ambiental sobre el agua, la tierra y las personas” $(9,10)$.

De acuerdo con la ENSin 2015, La Guajira ocupó el primer lugar en Colombia con una prevalencia de desnutrición global del 11\%, por encima de Chocó (6.3\%) y de Guainía (6.1\%), y para la desnutrición crónica superó la cifra nacional, pues mientras Colombia registró un 10.8\%, La Guajira reportó un 28.4\%(8). En el 2013 se registraron 19 niños fallecidos por dicha causa, distribuidos en los municipios de Albania, Dibulla, Maicao, Manaure, Riohacha, San Juan del Cesar y Uribia, aun cuando Manaure fue el municipio donde se concentraron un mayor número de casos (36.8\%), sin contar el alto porcentaje de subregistro que se presenta $(7,11,12)$.

Al rastrear los indicadores de situación alimentaria y nutricional de las madres gestantes y lactantes del territorio guajiro, se encuentra que, según un informe de la Procuraduría, el Instituto Colombiano de Bienestar Familiar reportó en su estudio de microfocalización que para el 2014 se encontraron 91 madres gestantes/lactantes en estado de desnutrición (13); entre tanto, la Defensoría del Pueblo manifestó que en ese mismo estudio se hallaron 253 madres gestantes y lactantes que requerían ser atendidas en salud y en nutrición (14).

Todo lo anterior se atribuyó, principalmente, a la escasez de agua potable en la región que, en los últimos años, se ha agudizado por una larga sequía, por la falta de disponibilidad de alimentos en el territorio y por los procesos de aculturación que han obligado a las comunidades adoptar prácticas alimenticias diferentes a las tradicionales $(14,15)$. Adicional a ello, es necesario mencionar las limitaciones del Estado para instaurar una política pública adecuada de atención a la población wayúu y la descoordinación entre los agentes estatales encargados de los programas de acción directa e indirecta relacionados con la seguridad alimentaria (5).

A todo esto se le adiciona que si bien esta problemática ha sido ampliamente abordada desde estudios epidemiológicos y biomédicos, han sido pocos los estudios publicados que abordan el fenómeno desde las voces de los afectados, en los que se tenga en cuenta la cosmovisión de dichas comunidades. Tal situación ha generado descontextualización e inefectividad en la implementación de los programas de asistencia alimentaria por parte del Estado (11,12). Por esta razón, la Organización Nacional Indígena de Colombia se ha pronunciado y ha exigido respuestas inmediatas a través de la concertación con los pueblos indígenas de las políticas, que "desde sus propias formas de vida, prevengan estas muertes y fortalezcan la vida sana, de acuerdo a sus propios planes de vida” (16).

Existen investigaciones que sugieren que la desnutrición infantil debe estudiarse desde la seguridad alimentaria, lo que implica incluir los aspectos socioculturales y socioambientales que caracterizan los sistemas alimentarios propios de cada comunidad, además de las prácticas culturales relacionadas con la alimentación, desde la recolección hasta su transformación, consumo, creencias, convicciones, gustos y prescripciones (16-18). Lo anterior supone, entonces, indagar sobre la cultura alimentaria que tejen las comunidades, pues esta, al ser 
entendida como "un producto de sinfín de decisiones a lo largo de su historia, que suponen clima, medio ambiente y capacidad de trabajo para aprovechar o no, los recursos disponibles para el grupo asentado en determinado sitio" (18), permitirá reconocer aquellos significados y procesos sociales vinculados a la alimentación, más allá de los enfoques exclusivamente dietéticos y nutricionales (19).

En una visita de algunos docentes de la Universidad Nacional de Colombia al Departamento de La Guajira quedó en claro que la producción y reproducción de la exclusión sistemática a la que se ha visto expuesta la comunidad indígena wayúu por parte de las entidades gubernamentales y académicas ha impedido comprender la problemática actual desde sus tradiciones y saberes ancestrales (20).

Estudios locales alrededor de la desnutrición infantil confirman que uno de los departamentos más afectados por la inseguridad alimentaria en Colombia es La Guajira $(13,14)$. Para el caso particular del municipio de Manaure, el Comité de Oxford de Ayuda contra el Hambre afirma que es un territorio muy vulnerable alimentariamente, dado que no solo no cuenta con las condiciones necesarias para la producción de alimentos, sino que "viven de los alimentos obtenidos en la frontera con Venezuela, generándose así una gran vulnerabilidad que determina que, el más leve cambio en el abastecimiento de alimentos y agua, desencadene una situación extrema de inseguridad alimentaria y nutricional” (21).

Basados en lo descrito y reconociendo la desnutrición infantil como un fenómeno multidimensional, este estudio enmarcó su análisis en el modelo teórico de la determinación social de la salud, desarrollado por Jaime Breilh, como una apuesta teórico-metodológica que busca explicar el proceso salud-enfermedad, reconociendo el carácter histórico y social que se encuentran inmersos en él, en contraposición a la explicación reduccionista y causalista que brinda el paradigma actual y dominante de la salud pública $(22,23)$. Para ello, Breilh propone analizar la relación entre la reproducción social, los modos de vivir y de enfermar y morir a partir de tres dimensiones: general, particular y singular $(22,23)$.

El propósito central de este estudio fue comprender las perspectivas comunitarias relacionadas con la desnutrición en niños de 0 a 7 años de edad en las comunidades wayúus Taiguaicat, Pañarrer y Limunaka del resguardo Manaure, en La Guajira. En la siguiente sección del artículo se presentan los lineamientos metodológicos que guiaron el estudio, los principales resultados y su respectiva discusión teórica.

\section{Desarrollo}

【 a pregunta de investigación que guió este estudio fue: ¿cuáles son las perspectivas y estra- tegias comunitarias relacionadas con la desnutrición en niños de 0 a 7 años en las comunidades wayúus, Taiguaicat, Pañarrer y Limunaka del resguardo Manaure, en La Guajira? 
Para responderla se llevó a cabo el proyecto de investigación Anaa Eirükü: perspectivas y estrategias comunitarias relacionadas con la desnutrición infantil (0-7 años), en tres comunidades wayúus del resguardo indígena Manaure, La Guajira, 2015-2016, con orientación metodológica de investigación participativa basada en la comunidad $(24,25)$. Este estudio cualitativo se desarrolló en dos momentos: el primero, denominado hermenéutico y comprensivo, pretendió develar los significados, las visiones y las perspectivas que reportan los miembros comunitarios alrededor del fenómeno de estudio. El segundo, denominado de acción o acompañamiento, se encargó de la formulación y ejecución de las estrategias comunitarias como alternativas de solución a la desnutrición infantil, y se está ejecutando actualmente.

El momento hermenéutico, que es el documentado en este manuscrito, se llevó a cabo en dos fases: una exploratoria y otra de recolección y análisis de la información. En la primera se realizó un acercamiento a la comunidad, a través de la consulta libre, previa e informada, para solicitar autorización y manifestar la intención de trabajar en el territorio. Así mismo, se desarrolló un diagnóstico rápido participativo (26), que permitió identificar las necesidades, las potencialidades y las limitaciones de la comunidad, con el fin de tener los insumos necesarios para tomar decisiones en la construcción conjunta del proyecto.

Posteriormente, y con la ayuda de la comunidad, se realizaron dos foros comunitarios, utilizando la técnica del árbol de problemas en la cual cada participante tuvo la oportunidad de expresar su opinión frente a las problemáticas que aquejaban a la población y cuál, desde su perspectiva, consideraba era la más urgente a investigar. En esta misma fase, la comunidad ayudó a definir todo lo relacionado con la selección de participantes, los instrumentos que se iban a utilizar y la forma como se recolectaría la información.

Para la segunda fase se consolidó un equipo de trabajo con actores clave de la comunidad, denominada coalición, con la metodología de investigación participativa basada en la comunidad, que tuviera el compromiso y el interés de ayudar en los diferentes momentos del proyecto $(24,25)$. Dicha coalición quedó conformada por las tres autoridades indígenas, el médico tradicional, la líder del clan, la coinvestigadora comunitaria, un padre indígena, una madre indígena y dos traductores.

Para recolectar la información se utilizaron tres técnicas: entrevistas semiestructuradas, foros comunitarios y fotovoz. Con las entrevistas semiestructuradas se pretendió un primer acercamiento a los participantes para identificar los conceptos, los significados ylas perspectivas que se tejen alrededor dela desnutrición infantil. Esta técnica se aplicó en los hogares delos participantes. Los criterios de inclusión para las 21 entrevistas se concertaron con las autoridades y la comunidad: a) miembro adulto (madre o padre indígena) perteneciente a una de las 21 familias indígenas, residente en una de las tres comunidades del estudio; b) con niños entre 0 y 7 años de edad; c) que se encontrara en la vivienda en el momento de la recolección, y d) que aceptara libremente participar en la investigación. 
Los foros comunitarios se usaron como una herramienta comunitaria que, además de facilitar la interacción entre los participantes, permitiera la identificación pública de sus opiniones, creencias, percepciones, intereses y actitudes alrededor de un objeto de estudio. Esto posibilitó profundizar en aspectos clave obtenidos en las entrevistas, así como la participación de más personas de la comunidad (27). Los criterios de inclusión fueron: a) miembro adulto de alguna de las 45 familias de las tres comunidades, b) que se encontrara en la comunidad en el momento de la recolección y c) que aceptaran libremente participar en la investigación.

La técnica de fotovoz - muy propia de la metodología de investigación participativa basada en la comunidad y ya previamente usada para abordar temas de salud en pueblos indígenas- se propuso con el fin de ampliar la información de los foros y las entrevistas; pero de una manera más visual y muy amigable con la comunidad. Se define como "una técnica innovadora de investigación participativa y comunitaria que en su origen permitió dar poder y voz, a través de la documentación de la realidad de su vida cotidiana mediante imágenes y narrativas, a aquellos que normalmente no son escuchados” (28). Para ello, se tuvieron en cuenta los mismos criterios de inclusión de los foros.

Inicialmente, se capacitó a las personas que participaron en el proyecto de manera que pudieran captar las imágenes, intentando dar respuesta a la guía que contenía cinco preguntas alrededor del buen vivir, la desnutrición infantil, sus causas, sus consecuencias y las soluciones que proponía cada uno de los participantes para esta. Una vez se tomaron las fotografías, se hizo una sesión con los participantes, donde cada uno explicó las razones por las cuales tomó cada una de las imágenes. ${ }^{2}$ La información de estas tres fuentes fue grabada y transcrita de forma literal posteriormente.

Para analizar la información se utilizaron las herramientas analíticas de la codificación y la categorización propuestas por la teoría fundamentada. Para este proceso se utilizó el software de análisis cualitativo Atlas.ti, versión 6.2, realizado en dos fases: codificación abierta, en la cual se explica cómo los fenómenos se relacionan entre sí y cuyo proceso derivó en la conformación de unas categorías descriptivas, y codificación axial, donde se reagruparon los datos analizados en la codificación abierta, en categorías analíticas que tenían relación entre sí (29). La codificación de cada uno de los testimonios se estructuró de la siguiente manera: rol del participantecomunidad-técnica utilizada-género-consecutivo.

Respecto a las consideraciones éticas, este proyecto contó con el aval del Comité de Ética de la institución académica que lideró el proyecto y se basó en las normas que regulan la ética de la investigación en Colombia (30) - y en el mundo (31), las pautas del Consejo de Organizaciones

2 Las preguntas fueron: ¿qué elementos o cosas representan para ti bienestar-buen vivir? ¿Qué elementos o cosas representan la desnutrición infantil? ¿Qué elementos o cosas crees que causan la desnutrición infantil? ¿Qué consecuencias tiene en los niños la desnutrición? ¿Cuáles creen son las soluciones a la desnutrición infantil? 
Internacionales de Ciencias Médicas de 2002 (32) — y los requisitos éticos para la conducción responsable de la investigación clínica (33) y en el contexto de pueblos indígenas (34). Los resultados se estructuraron alrededor de dos categorías analíticas identificadas en el estudio, las cuales dan cuenta de las perspectivas comunitarias alrededor de la desnutrición infantil que refirieron los participantes y que se describen a continuación.

\section{Las prácticas arijunas: de la pérdida de lo ancestral a la enfermedad del hambre}

Los participantes consideran que el contexto actual del territorio wayúu es diferente al que tenían, ya que tiempo atrás contaban con las condiciones suficientes para pastorear y proveer a sus familias de los alimentos necesarios para la subsistencia, situación que hoy no se tiene: "Los indígenas antes no daban a conocer necesidades de este tipo, porque antes ellos tenían sus animales donde podían ordeñar y estar pendiente de ellos, si se enfermaban no era por alimentación como hoy en día pasa” (autoridad indígena-P-EV1-M-54). Véase figura 1.

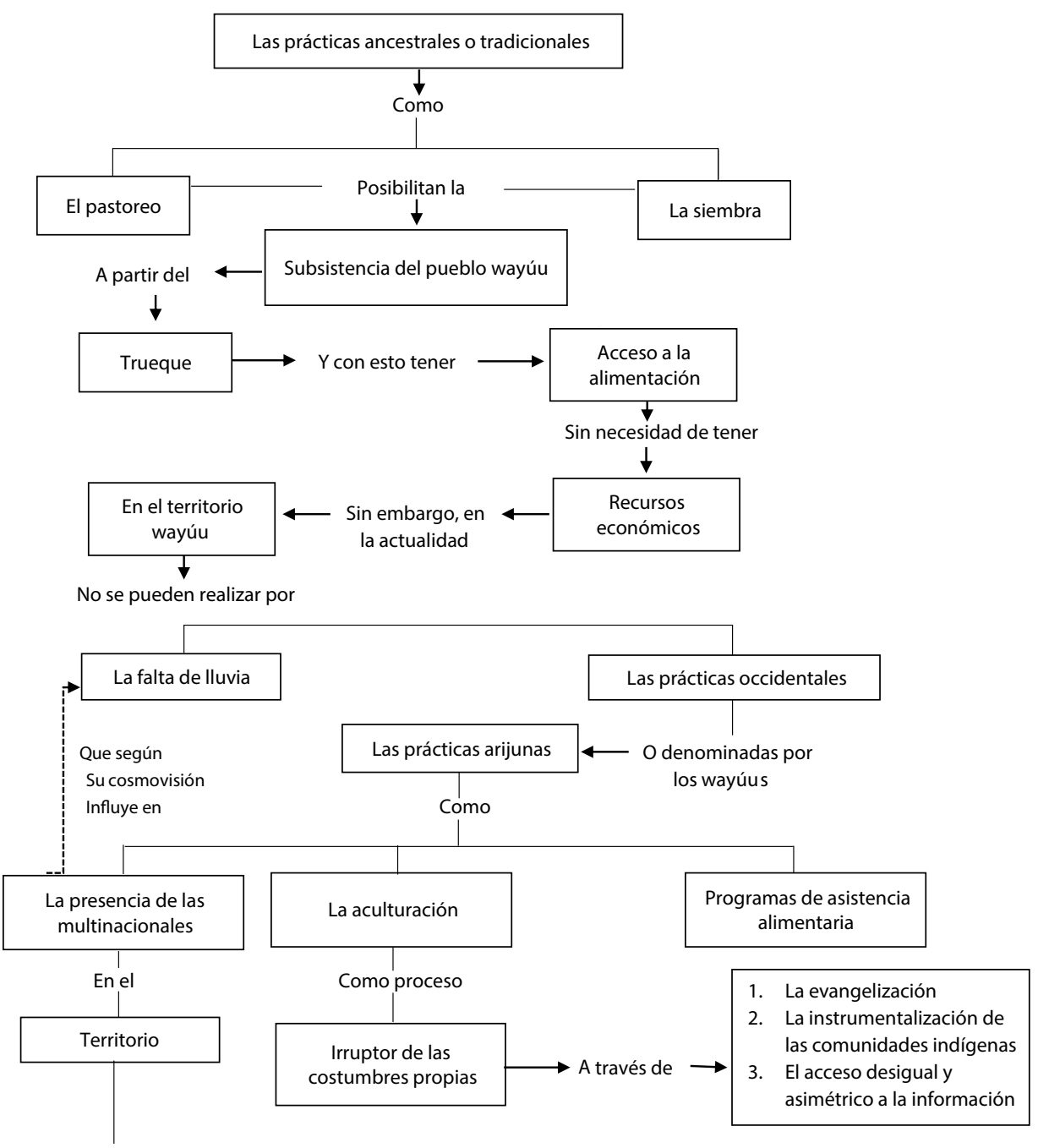




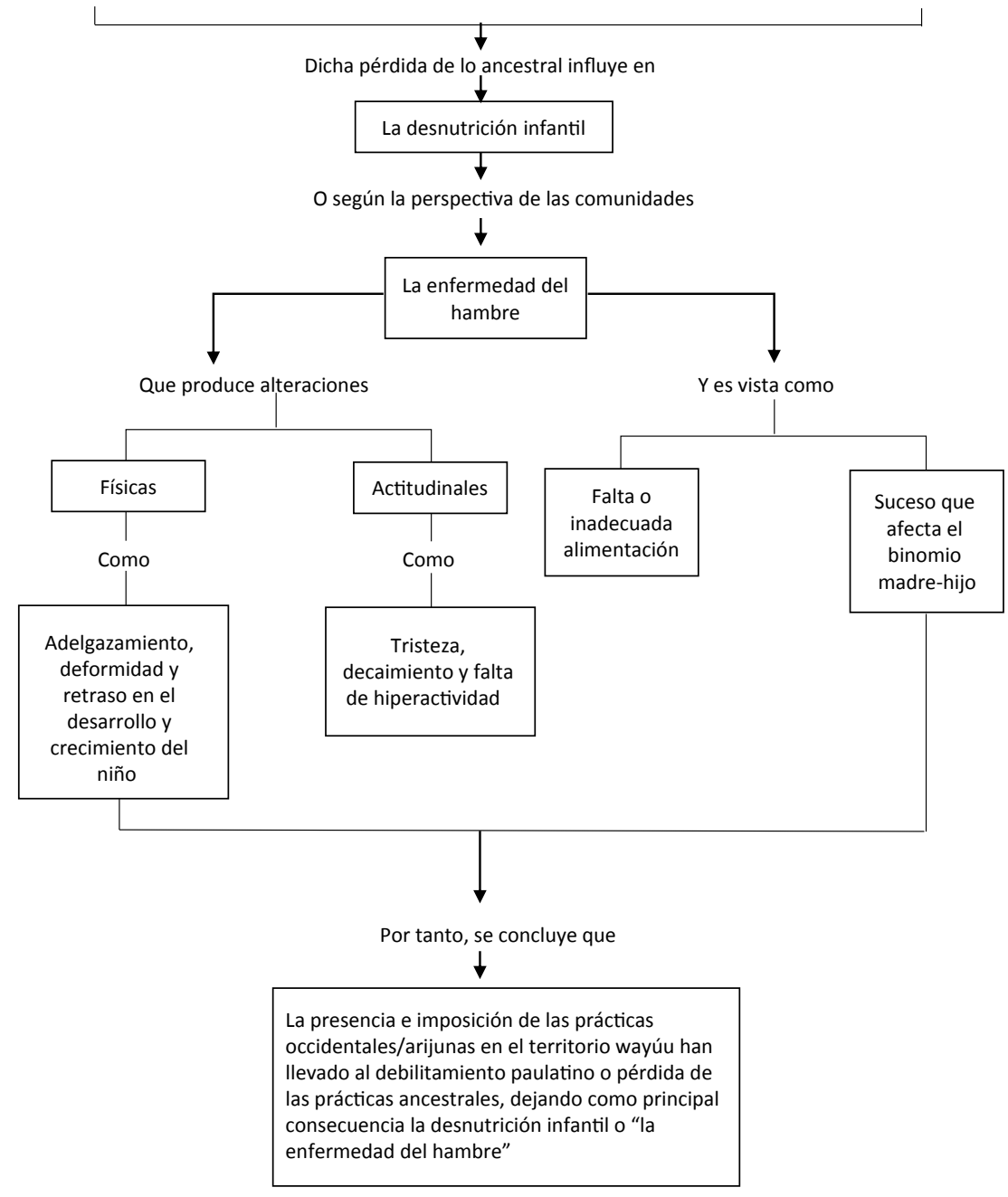

Figura 1. Mapa conceptual de la categoría analítica "las prácticas arijunas: de la pérdida de lo ancestral a la enfermedad del hambre"

Antes, las prácticas ancestrales como el pastoreo y la siembra formaban parte del diario vivir, porque se valían de sus sembrados, de los frutos propios de la región y de los animales como principal fuente de subsistencia. Esto se hacía no solo para alimentar a los niños, sino también para realizar una práctica muy común en sus ancestros, denominada trueque, la cual permitía intercambiar con otras familias gran variedad de víveres, sin necesidad de tener recursos económicos:

Nosotros accedemos por medio de trueque, yo tengo chivo y lo cambio por paca de fríjol, paca de maíz, lo que tenga el otro clan, hoy en día ya toca mirar a ver cómo poder tener recursos monetarios de los cuales se puedan intercambiar y hacer la compra. (Madre indígena T-EV10-F-13)

Al indagar respecto a la razón por la cual habían perdido dichas tradiciones, uno de los elementos que emerge en sus diferentes narrativas es la falta de lluvia: "En mi cosmovisión es porque no hay lluvia, porque si tuviéramos cómo poder sembrar, cómo poder hacer las 
prácticas anteriores que uno venía haciendo, que era sembrar, recolectar los granos, guardarlos durante el periodo de sequía” (Madre indígena T-EV7-F-25).

Sin embargo, desde su cosmovisión, dicha condición ambiental está influenciada principalmente por dos prácticas arijunas. Una de ellas es la práctica extractiva por parte de multinacionales, por cuanto estas empresas se perciben como invasivas, irrespetuosas, dañinas y poco claras en el momento de expresar su intención en la zona: "Ellos están sobre nosotros porque así lo hemos permitido, ya que somos mayoría y aun así callamos; además nunca para mí, fueron claros, no entendí cuál era el propósito, ni mucho menos sus consecuencias" (Padre indígena T-FC1-M1-31).

Adicional a ello, algunos expresaron la falta de responsabilidad social y ambiental que subyace el actuar de las multinacionales, a través de los imaginarios que tienen alrededor de la influencia de estas en el cambio climático, que actualmente padece su territorio. Según sus narraciones, dichas instituciones modifican el clima con el sobrevuelo de sus avionetas sobre el territorio wayúu, porque no les conviene que llueva:

Es que nosotros hemos visto que un helicóptero o una avioneta desplaza la lluvia, porque cada vez que va a llover, pasa ese avión y la desplaza, ya no llueve... Porque la multinacional desvía la lluvia para que el gaseoducto no se inunde, ellos fumigan en toda la zona del gaseoducto. (Madre indígena L-EV14-F-22)

La segunda práctica arijuna que influencia la pérdida de lo ancestral es la aculturación, ${ }^{3}$ como proceso que irrumpe las costumbres propias del pueblo y donde lo que prima es la imposición de la cultura occidental, a partir de:

a) Los procesos de evangelización en los wayúus, que los lleva a naturalizar la situación de escasez, pobreza y desigualdad a la que se ven enfrentados, bien sea porque es un "castigo divino" por sus pecados o porque el ser humano debe pasar hambre y sed para lograr "la gloria eterna":

Lo que digo es que como se nos ha evangelizado, se nos mostró la Biblia y explicando desde ahí que todo esto tiene que pasar para la venia del único Dios supremo, a lo que hoy me sujeto es a la palabra, a eso que me manda el Dios de todos... Que debemos de pasar por esto, padecer de cosas vitales como ser humano como hambre y sed, para ver la gloria eterna. (Padre indígena T-FC1-M1-75)

b) La instrumentalización que padecen los indígenas de esta zona a manos de investigadores 0 de particulares, que llegan al territorio con supuestos deseos de ayudar; pero que en el fondo se aprovechan de su condición de superioridad y de la situación actual desfavorable que

3 Para este estudio se tomó la definición de aculturación de Breilh y Tilleria, que la definen como "proceso de adaptación (forzoso la mayoría de veces) de las diversas etnias que, debido a la situación geográfica o las circunstancias históricas, políticas o económicas han tenido que convivir, impuestas por un grupo dominante, enmarcado casi siempre, en un modelo neoliberal que lleva al deterioro de los modos de vivir de esas poblaciones" (35). 
viven las comunidades: "El acercamiento por intereses particulares de ellos mismos, por sus explotaciones bajo nuestro desconocimiento e ignorancia de las cosas y consecuencias de las supuestas ayudas, las cuales han sido para perjudicarnos" (Autoridad indígena P-FC2-M1-13).

c) El acceso desigual y asimétrico a la información que tienen las comunidades indígenas, respecto al que tienen los occidentales, caracterizado principalmente por las barreras idiomáticas o por la poca posibilidad de acceder a diferentes medios de comunicación, para estar actualizados de lo que pasa en el interior y por fuera de su territorio. "Esto pasa porque no sabemos el español, porque no sabemos qué pasa afuera, porque hace falta que un wayúu nos explique lo que hay a nuestro alrededor y el cómo debemos realizar las cosas" (Padre indígena T-FC1-M1-34).

d) Los programas de asistencia alimentaria brindados por el Gobierno, que se venden como una alternativa de solución ante la problemática de escasez de alimentos y de agua. Sin embargo, muchos de ellos no llegan a todas las comunidades y generan expectativas, engaños y esperanza de que las ayudas llegarán pronto a la comunidad. "He escuchado que se les puso a comunidades vecinas tanques para almacenar agua, de ahí que, a nosotros, estas comunidades a las cuales hoy preguntas, no se ha generado ningún tipo de beneficios, nuestra situación es crítica” (Padre indígena P-FC2-M1-17).

En ese sentido, para los participantes del estudio, las prácticas occidentales han influido en la pérdida de sus prácticas ancestrales y ello ha traído como consecuencia la desnutrición infantil que padecen actualmente como pueblo.

Dicha enfermedad es considerada por algunos miembros de la comunidad la enfermedad del hambre, pues encuentran una estrecha relación entre la falta de alimentos y la imposibilidad de brindarles a los niños lo necesario para su sano desarrollo, de manera oportuna y con la cantidad adecuada:

La desnutrición en los niños es por aguantar hambre, porque uno no tiene nada para darles... Aunque también es que uno tiende a darle poquito para que no se acabe lo que se tiene, entonces un sorbo de alimento al día no es suficiente para recargar la energía, para que el niño se divierta, juegue, para que haga todas las actividades que conciernen a la niñez. (Madre indígena L-EV12-F-18)

Es no comer a tiempo, es cuando existe desorden para los alimentos, por ejemplo, que un día aguantas hambre y al otro coma, en muchas ocasiones nos hace daño estomacal... Y por eso les da eso que tú llamas desnutrición. (Madre indígena L-EV19-F-11)

Para otros es una enfermedad que produce alteraciones físicas o anormalidades, reconocidas sobre todo por las condiciones o el estado físico, como el adelgazamiento de los niños, o actitudinales, como la tristeza, el decaimiento y la falta de hiperactividad, que impiden el desarrollo normal del niño. "Da mucho pesar porque son decaídos, muy tristes, no actúan como un niño normal, es decir, hiperactivo, colaborador y trabajador. Es que eso es notable, 
este estado de los niños enfermos es visible, sus extremidades superiores y el tronco" (Padre indígena T-FC1-M1-40).

Así mismo, algunos participantes consideran que la desnutrición se da en los niños por la mala alimentación a la que estos acceden: "Es que si solo se les da chicha (bebida ancestral de los wayúus a base de maíz), tienden a tener una barriguita redonda y grande y todo su cuerpo es delgadito” (Madre indígena L-EV14-F-24).

\section{La enfermedad del hambre: su relación con la alimentación del binomio madre-hijo, durante la gestación y después de esta}

En esta categoría se identificó que la enfermedad del hambre no es un suceso repentino que aparece durante la etapa de la niñez, sino que comienza mucho antes, específicamente con la gestación o, en su defecto, con las condiciones anteriores de salud de la madre. En este sentido, algunos participantes encuentran que hay niños que desde el vientre están en condiciones alimentarias desfavorables, lo cual obedece, en la mayoría de ocasiones, a que su madre no pudo alimentarse adecuadamente (figura 2).

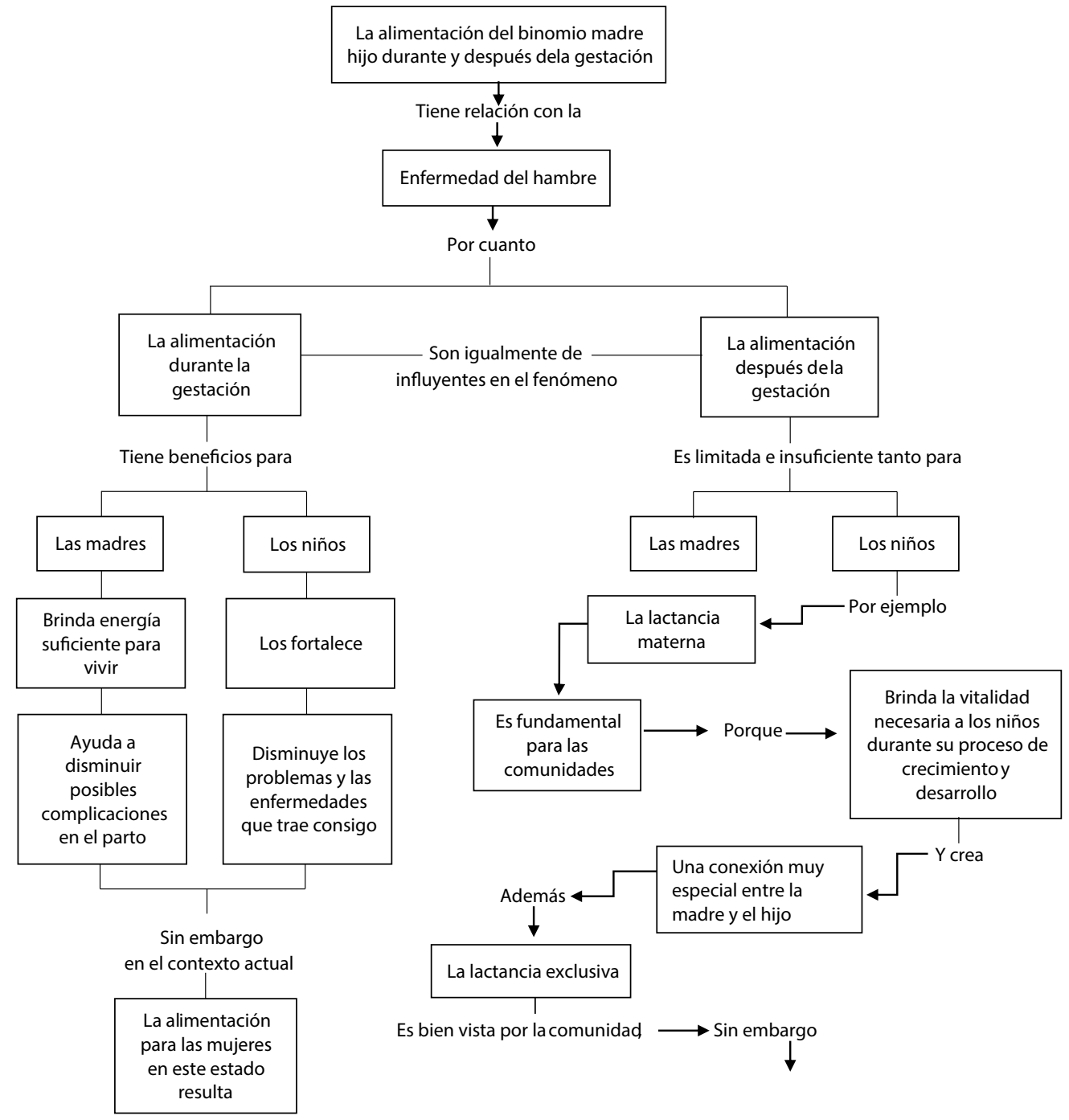




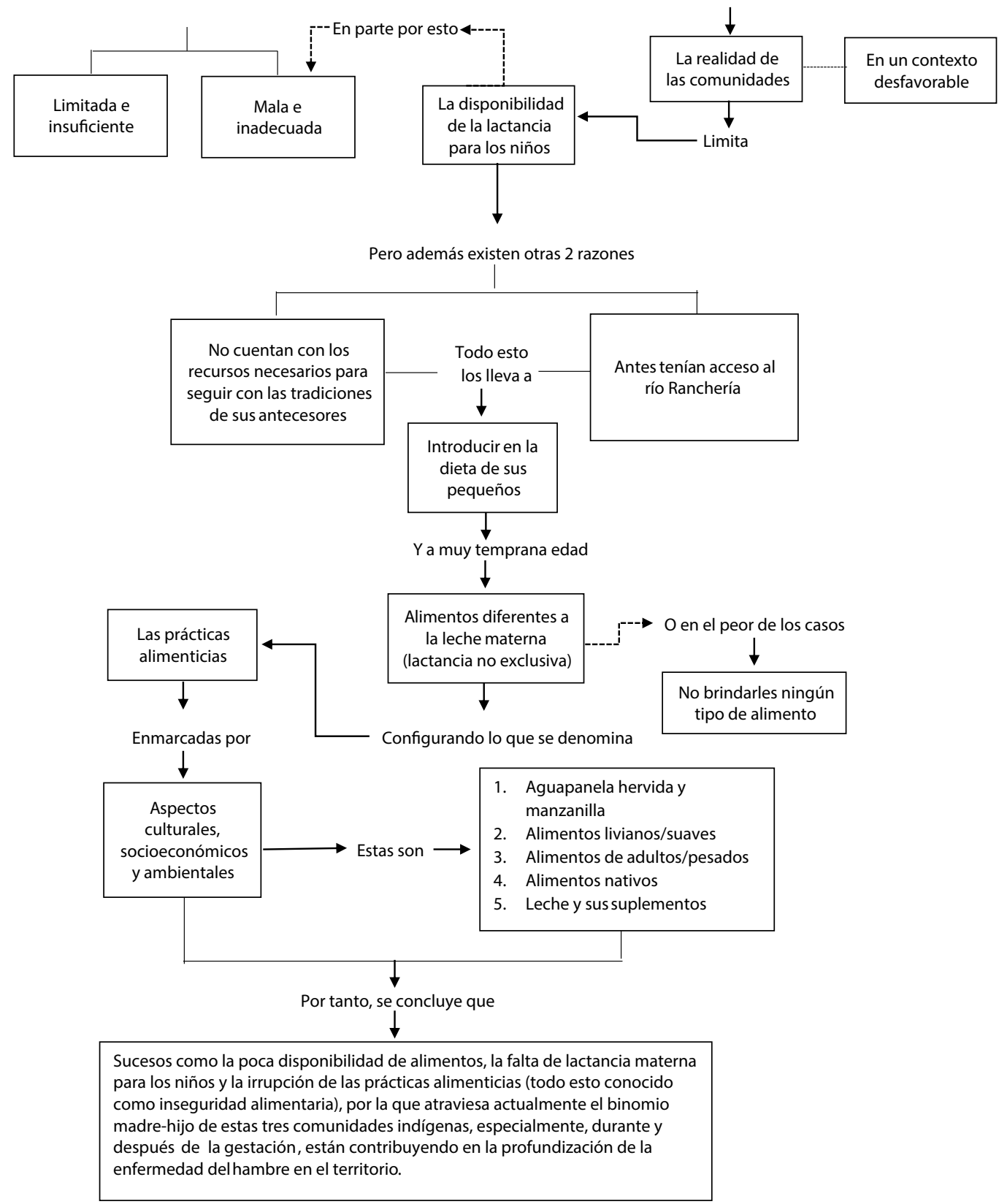

Figura 2. Mapa conceptual de la categoría analítica "la enfermedad del hambre: su relación con la alimentación del binomio madre-hijo, durante la gestación y después de esta"

Esto, según los participantes, afecta el relacionamiento en el interior del binomio madrehijo: “Ella no tiene fuerzas, está enferma y es porque hay hambre, no tiene cómo alimentarse, no hay nada y aparte de eso no tiene plata... Y si ella no tiene fuerza y está desnutrida, sus hijos también lo estarán” (Madre indígena FV9-L-P3-70).

Al indagar en los participantes por los beneficios que tiene la alimentación para las mujeres durante la gestación, ellos afirmaron que esta les brinda energía suficiente para vivir, disminuye las posibilidades de complicaciones en el parto, fortalece al niño y reduce los problemas o las enfermedades con las que este pueda venir. Sin embargo, de acuerdo con 
el contexto actual que viven las tres comunidades, se encuentra que los diferentes actores perciben como limitada e insuficiente la alimentación de las mujeres en ese estado. Esto se presenta por la dificultad que tienen para acceder a los víveres, entre otras razones, por las condiciones socioeconómicas a las que se ven enfrentados diariamente, la cual los lleva a consumir solo lo que se consiga.

Respecto a la alimentación disponible, en especial para los niños, una vez las madres han dado a luz, se encuentra que, pese a que tejen significados de importancia y de beneficios alrededor de la lactancia por los múltiples beneficios y bondades que trae para el desarrollo oportuno de sus niños, su situación fáctica es otra. De esta manera, encuentran dificultades para brindársela a los niños por tres razones relacionadas con la poca disponibilidad de alimentos, la falta de acceso al agua potable y la poca disponibilidad para lactar.

En particular, las comunidades no cuentan con los recursos necesarios para seguir con las prácticas de subsistencia de sus ancestros, lo cual disminuye las posibilidades de que una madre pueda acceder a una buena alimentación, y ello le limita su disponibilidad de leche materna: "Yo te cuento que no hemos podido seguir el legado de nuestros antepasados porque no tenemos cómo mantener a los niños porque, ya las cosas... Es más complicada, es que están pasando trabajo (dificultades) y eso no significa que no los queramos” (Padre indígena L-EV17-M-01).

El segundo motivo es porque antes esta comunidad tenía acceso al río Ranchería, el cual, aunque quedase lejos, les posibilitaba preparar alimentos para todos, en especial para las mujeres durante su gestación, lo cual potenciaba que tuvieran disponible leche materna para los bebés: "Nosotros los varones debíamos buscarlos por la tarde y salir con ellos al día siguiente en la madrugada, como a las 2:00 a.m., para poder rendir en el acarreo del agua y así hacíamos nosotros y todos los wayúus anteriormente, y éramos felices y estábamos bien alimentados" (Médico tradicional P-FC2-M1-29). Todo esto los ha llevado a introducir en la dieta de sus hijos alimentos diferentes a la leche materna (lactancia no exclusiva) desde muy temprana edad:

Durante los primeros 6 meses darle teta, pero como a mí no me baja, entonces yo empiezo a darle chicha antes de los 6 meses... Es que la leche es indispensable. Mire que cuando un ternero toma la leche de su madre, es gordito y muy bonito, así mismo son los bebés necesitan la lactancia. (Madre indígena P-EV3-F-29)

Incluso, en algunas situaciones extremas, se ven obligados a no brindarles ningún tipo de alimento, porque no tienen con qué, realidad que es difícil de explicar a los niños:

Por la noche, mis dos muchachos, los más pequeños, porque los que ya pasaron por eso, ellos lloraban mucho por la noche, entonces me tocaba coger, hervir, hacer mazamorra, el clarito, echarle un poquito de azúcar y darles a ellos eso, si había, si no, no les daba nada, porque en realidad nunca me bajaba la leche. (Madre indígena T-EV8-F-16) 


\section{Discusión}

Comprender las perspectivas comunitarias alrededor de la desnutrición infantil en el territorio wayúu, implica reconocer que las condiciones que enfrenta diariamente dicho pueblo son el resultado de una larga historia que los ha determinado, forjada por procesos de presión social, cultural, económica y política que han configurado la crisis humanitaria que hoy afronta $(20,35,36)$. En este apartado se discuten los tres hallazgos más relevantes que se evidenciaron en esta investigación con relación al fenómeno de estudio.

De acuerdo con la narrativa de los participantes, el primer hallazgo está relacionado con los aspectos que mejor explican la pérdida paulatina de sus prácticas ancestrales, y con ello la desnutrición de sus niños y niñas. Para los entrevistados, dicha pérdida se explica a partir de las prácticas occidentales o arijunas que se están llevando a cabo en su territorio, entre las cuales destacan la presencia de multinacionales que, en una lógica mercantil, se han instalado de manera dominante; el proceso de aculturación, como un asunto irruptor de sus prácticas autóctonas, y el asistencialismo (descontextualizado e inefectivo) del Estado en algunas rancherías.

Justamente, ese hallazgo coincide con lo expresado por Lander, cuando afirma que esa pérdida de lo ancestral está signada por una idea desarticulada de progreso, donde el extractivismo ha hecho su nido y la dinámica civilizatoria se ha transformado en una práctica destructora (37). Además, las expectativas de la modernidad, como la abundancia, la libertad y la felicidad, han fracasado al entender el capitalismo, la racionalidad instrumental y el espíritu egoísta como sinónimos de sociedad moderna (38).

De acuerdo con autores como Natalia Orduz y Felipe Rodríguez, la desviación del río Ranchería, a manos de la multinacional Cerrejón, trajo efectos desde afectaciones al ciclo hidrológico natural de la zona hasta hambre, miseria y devastación para sus comunidades indígenas, principalmente $(39,40)$. En ello coinciden con Restrepo et al., quienes afirman que existe una estrecha asociación entre el estado nutricional de los niños de una comunidad indígena de Colombia y las condiciones socioeconómicas y de pobreza que enfrentan sus familias, como son para el caso de los embera-katíos del Departamento de Córdoba (Colombia) la tala indiscriminada de árboles para el comercio; la sustitución por cultivos ilícitos, que lleva la disminución de la caza, y la construcción de la hidroeléctrica, que disminuyó considerablemente la pesca del bocachico (41).

Relacionado con lo anterior, se encontró que en el Departamento del Huila (Colombia), el proyecto de una hidroeléctrica, además de traer consecuencias irreversibles para el ambiente, como la pérdida de la biodiversidad, trajo consecuencias sociales, como la pérdida de prácticas culturales ancestrales relacionadas con la pesca y el cultivo de especies, como el cacao, que "reprimen los derechos soberanos de los campesinos sobre las tierras que han ocupado por generaciones” (42). 
Desde una perspectiva analítica de determinación social de la salud, lo expuesto pone de manifiesto que la presencia del fenómeno de la desnutrición infantil en las tres comunidades no es más que un proceso histórico y complejo que ha estado enmarcado por condiciones de vulnerabilidad económica, política, social, ideológica y cultural, que han venido configurándolo y determinándolo poco a poco; además, provocan transformaciones profundas en los modos de vivir, de enfermar y de morir de los wayúus $(22,23,35)$. De acuerdo con Breilh, a partir del modelo actual civilizatorio, enmarcado en una lógica de estructuras de poder que conllevan una dominación histórica y colonización cultural, pérdida de derechos, exclusión, explotación de todo tipo de recursos y desigualdades evitables, perpetúa la aceleración del ritmo de producción capital en la economía global y deteriora la vida del planeta y de las sociedades actuales (35).

Frente a los procesos de aculturación del pueblo wayúu, la Gobernación de La Guajira, en su Plan de Desarrollo 2016-2019, hacía un llamado a la reflexión, donde cuestiona si es justo y válido llamar "salvajes" a las personas que han habitado milenariamente el territorio que han propendido a proteger la naturaleza y las especies que los rodean, que saben trabajar en comunidad y que han sido exterminados y maltratados históricamente por la sociedad que se autodenomina "civilizada" (43).

Muchos hablan de las barreras culturales como las culpables de que los indígenas no avancen hacia el desarrollo, pero la gran verdad es que los indígenas no tienen barreras, las barreras las impone el mundo occidental, al pretender replicar y reproducir esquemas ortodoxos y rígidos que han resultado fructuosos solo en otro tipo de población(43).

Lo anterior, sumado al asistencialismo descontextualizado del Estado, llega a algunos lugares cercanos del casco urbano de La Guajira y su ausencia en otros tramos del territorio, más adentrados y lejanos de la ciudad, situación que demuestra desconocimiento, la falta de políticas claras y proyectos efectivos, encaminados a dar soluciones estructurales a diferentes desafíos de la región, como la garantía del agua, la alimentación, la salud y la educación $(5,11,12,14)$.

El segundo hallazgo está relacionado con las condiciones alimentarias del binomio madre-hijo, y es que para los participantes la enfermedad del hambre desarrollada por el niño comienza desde mucho antes de su nacimiento y no solo lo afecta a él, sino a la madre, cuando esta no contó con las condiciones necesarias para alimentarse durante su proceso de gestación.

Esto guarda relación con lo expresado en la Declaración de Atitlán, en la que se afirma que la desnutrición infantil está ligada a las condiciones alimenticias del binomio madre-hijo, durante la gestación y después de esta, en aspectos como la poca disponibilidad de alimentos, la falta de lactancia para los niños y la incorporación temprana de víveres diferentes a la leche materna. Esta situación pone de manifiesto la necesidad urgente de que el Gobierno garantice la seguridad y la soberanía alimentaria a los pueblos indígenas del país (44). 
La salud materno-infantil siempre ha sido una prioridad presente en las agendas internacionales y en todos los países del mundo, y se ha constituido no solo en un indicador que determina el desarrollo socioeconómico de una nación, sino también en un asunto ético social, en un derecho humano básico $(16,45)$.

Según el estándar internacional establecido por la Organización Mundial de la Salud, la lactancia exclusiva debe ser durante los seis primeros meses de vida(46). Sin embargo, para las comunidades indígenas participantes en esta investigación, debería ser durante todo el primer año de vida. Esto coincide con otros estudios, que afirman que el ideal de lactancia exclusiva en las comunidades indígenas es superior al de poblaciones no indígenas del país, que oscila entre 12 y 24 meses (47).

Finalmente, el tercer hallazgo está relacionado con la importancia que representa para los participantes wayúus de esta investigación tener acceso a sus prácticas alimentarias ancestrales. Según lo expresan, los alimentos tradicionales evocan grandes y gratos recuerdos para ellos, considerados por la gran mayoría como buenos, sanos e ideales, caso contrario a lo que piensan de los occidentales, que son nocivos y perjudiciales para sus procesos de crecimiento y desarrollo; pero que, al no tener la posibilidad de acceder a los propios, deben incorporarlos en sus prácticas alimenticias.

Esto guarda relación con lo expresado por el antropólogo Mintz (48) y otros investigadores del tema $(14,18,19)$, cuando afirman que la alimentación como proceso cargado de historicidad reconoce que los víveres que consume un colectivo tienen narraciones asociadas con su pasado; historias que los individuos construyen a partir de emociones y recuerdos, en cuanto a la manera de encontrarlos, recolectarlos, procesarlos, prepararlos, servirlos y consumirlos; que no son comidos simplemente, sino que "son portadores de sentido y este sentido les permite ejercer efectos simbólicos, reales, individuales y sociales” (48).

Estudiosos de la determinación social de la salud, como Breilh $(22,23)$, y de la determinación social de la alimentación-nutrición, como Rivera (49), hacen un llamado a entender el proceso producción-consumo de alimentos, más allá del paradigma reduccionista que hasta ahora se ha empleado para su comprensión. Es necesario no solo considerar la dimensión biológica de la alimentación, sino también sus dimensiones sociales y culturales (49), pues es evidente que el acceso y el consumo de los grupos poblaciones es socialmente diferenciado, dado que dependen en gran medida de su capacidad adquisitiva $(22,23,49,50)$.

Teniendo en cuenta lo anterior, desde las perspectivas comunitarias de los wayúus, la desnutrición infantil es un fenómeno complejo, multidimensional, histórico que se ha venido configurando a lo largo del tiempo, bajo la lógica nociva de un modelo económico neoliberal, salvaje, acumulativo y extractivista que profundiza unas relaciones de poder injustas y desiguales, cada vez más arraigadas y “naturalizadas” en Colombia, especialmente afectando a las comunidades indígenas del país. 
En ese mismo sentido, es claro que el acercamiento de los occidentales al territorio wayúu ha debilitado las prácticas alimenticias tradicionales en el interior de las comunidades, lo que ha generado alta dependencia de alimentos a los cuales, por las lógicas del mercado actual, tampoco pueden acceder por sus altos costos y su falta de capacidad adquisitiva como individuos o familias.

Este estudio representa un aporte significativo a la comprensión de la desnutrición infantil wayúu desde las perspectivas de los miembros comunitarios; sin embargo, una limitación encontrada en el estudio fue no haber contado con recursos suficientes para haber logrado una mayor cobertura de participantes, y aunque no era el propósito del estudio, para haber generado la línea de base nutricional de los niños existentes en la comunidad para la identificación de niños desnutridos o en riesgo de padecer dicha enfermedad.

A partir de los resultados encontrados, se hace necesario avanzar en nuevas investigaciones que aborden el estado actual de la comunidad y su dieta básica, con el fin de determinar a profundidad las condiciones nutricionales y el uso de productos propios para promover su seguridad alimentaria. Así mismo, estudios enfocados en generar alternativas interculturales que permitan promover la autonomía wayúu y el rescate de sus valores, creencias y saberes como nación indígena en lo relacionado con la nutrición, pero en diálogo con las condiciones reales actuales dentro de sus comunidades y con las dinámicas de los territorios que habitan.

\section{Contribución de los autores}

Tennifer Marcela López-Ríos: su aporte estuvo encaminado a la formulación del proyecto de investigación, la recolección de los datos, el análisis e interpretación de la información. Así mismo, contribuyó en la dirección y liderazgo de la escritura del artículo y en la revisión crítica tanto del contenido sustancial por plasmarse en el artículo como en la versión aceptada para publicar.

Sergio Cristancho: su aporte estuvo encaminado a la revisión de la formulación del proyecto de investigación, apoyo en el análisis e interpretación de la información. Así mismo, contribuyó en la escritura del artículo y en la revisión crítica de la versión aceptada para publicar.

Isabel Cristina Posada-Zapata: su aporte estuvo encaminado a apoyar el análisis e interpretación de la información. Así mismo, contribuyó en la escritura del artículo y en la revisión crítica de la versión aceptada para publicar. 


\section{Fuente de financiación}

E sta publicación es resultado de una investigación realizada con el apoyo financiero del

EFondo de Apoyo Docente de la Facultad Nacional de Salud Pública de la Universidad de Antioquia y la Estrategia de Sostenibilidad del Comité para el Desarrollo de la Investigación (CodI) de la misma universidad.

\section{Conflicto de intereses}

L os autores declaran no tener ningún conflicto de intereses y que los puntos de vista expresados en el artículo son responsabilidad de ellos y no de la institución en la que trabajan o de la fuente de financiación.

\section{Referencias}

1. Organización de las Naciones Unidas para la Alimentación y la Agricultura (fAO). Panorama de la Seguridad Alimentaria y Nutricional en América Latina y el Caribe 2013. Hambre en América Latina y el Caribe: acercándose a los Objetivos del Milenio. Roma: Editorial FAO; 2014.

2. Fondo de las Naciones Unidas para la Infancia (Unicef). Mejorar la nutrición infantil: El imperativo para el progreso mundial que es posible lograr. Nueva York: Editorial Unicef; 2013.

3. United Nations Organization. State of the world's indigenous peoples. New York: United Nations Publication; 2009.

4. Fondo de las Naciones Unidas para la Infancia (Unicef). La desnutrición infantil: causas, consecuencias y estrategias para su prevención y tratamiento. Madrid: Editorial Unicef; 2011.

5. Organización de las Naciones Unidas para la Alimentación y la Agricultura (fAO), Departamento Administrativo para la Prosperidad Social (DPS). Comida, territorio y memoria: situación alimentaria de los pueblos indígenas colombianos. Bogotá: fAO; 2015.

6. Comisión Económica para América Latina y el Caribe (Cepal). El costo del hambre: impacto económica y social de la desnutrición infantil. Panorama general. Naciones Unidas: Santiago de Chile; 2007.

7. Noreña-Herrera C, Leyva-Flores R, Palacio-Mejía LS, Duarte-Gómez MB. Ethnic disparities in the use of reproductive health services by indigenous and African-descendant Colombian women. Cad Saúde Pública. 2015;31(12):2635-48.

8. Ministerio de la Protección Social, Social, Instituto Nacional de Salud, Instituto Colombiano de Bienestar Familiar (IсBғ), Profamilia. Encuesta Nacional de la Situación Nutricional en Colombia (ENSIN) 2015. Bogotá: ICBF; 2016. 
9. Organización Nacional Indígena de Colombia. Pueblo wayúu [internet]. [Citado 2020 dic 20]. Disponible en: https://www.onic.org.co/pueblos/1156-wayúu

10. Ministerio de Cultura de Colombia. Wayúu: gente de arena, sol y viento [internet]. [Citado 2020 dic 20]. Disponible en: https://www.mincultura.gov.co/prensa/noticias/Documents/ Poblaciones/PUEBLO\%20WAY\%C3\%9AU.pdf

11. Colombia, Gobernación de La Guajira, Departamento Administrativo de Planeación de La Guajira. Evaluación y seguimiento morbi-mortalidad y bajo peso al nacer por desnutrición, Departamento de La Guajira [internet]. [Citado 2018 jun 18]. Disponible en: http://www.laguajira.gov.co/web/attachments/1731_MORBI\%20MORTALIDAD\%20 INFANTIL\%20DPTO\%20DE\%20LA\%20GUAJIRA.pdf

12. Colombia, Gobernación de La Guajira, Secretaría Departamental de Salud. Análisis de la situación de salud del Departamento de La Guajira [internet]; 2011 [citado 2018 jun 18]. Disponible en: https://www.minsalud.gov.co/plandecenal/Paginas/mapa/Analisisde-Situacion-Salud-LaGuajira-2011.pdf

13. Colombia, Procuraduría General de la Nación. La Guajira: pueblo wayúu, con hambre de dignidad, sed de justicia y otras necesidades insatisfechas. Bogotá; 2016.

14. Colombia, Defensoría del Pueblo. Crisis humanitaria en La Guajira 2014: acción integral de la Defensoría del Pueblo en el departamento. Bogotá; 2014.

15. Duarte M, Ortega M, Mora L, Coromoto N. Un modelo de atención en salud al pueblo wayúu en la frontera colombo-venezolana. Rev Panam Salud Pública. 2011;30(3):272-8.

16. Calderón Farfán JC. Perspectivas comunitarias de las condiciones socioculturales de salud infantil del resguardo indígena Huila: municipio de Iquira (H). 2013-2014. [informe de investigación para optar al título de Magíster en Salud Pública]. Medellín: Convenio Universidad de Antioquia-Universidad del Tolima; 2014.

17. Gobernación de Antioquia, Mejoramiento Alimentario y Nutricional de Antioquia (maNA), Universidad de Antioquia-Escuela de Nutrición y Dietética. Una historia triste, pero con un final feliz: sistematización, formación en hábitos alimentarios saludables y derechos y deberes del sistema de seguridad social en salud en 30 municipios del departamento. Medellín: Gobernación de Antioquia; 2003.

18. Camou E. Nutrir la persona, nutrir la identidad: reflexiones filosóficas sobre antropología y cultura alimentaria. En: Sandoval S, Meléndez J. Cultura y seguridad alimentaria: enfoques conceptuales, contexto global y experiencias locales. México: Plaza y Valdés; 2008. p. 29 у 30.

19. Del Pino P, Mena M, Torrejón S, Del Pino E, Aronés M, Portugal T. Repensar la desnutrición: infancia, alimentación y cultura en Ayacucho, Perú. Lima: Acción Contra el Hambre-Instituto de Estudios Peruanos; 2012.

20. Hernández M, Pachón X. Informe de la visita al Departamento de La Guajira entre los días 4 y 5 de febrero de 2016. Universidad Nacional de Colombia, Sede Bogotá; 2016.

21. Comité de Oxford de Ayuda contra el Hambre. Mapeo de la situación de los medios de vida y la seguridad alimentaria de las familias vulnerables en la Alta Guajira [internet]. [Citado 2020 dic 18]. Disponible en: https://www.humanitarianresponse.info/es/ operations/colombia/document/mapeo-de-la-situaci\%C3\%B3n-de-los-medios-de-viday-la-seguridad-alimentaria 
22. Breilh J. La determinación social de la salud como herramienta de transformación hacia una nueva salud pública (salud colectiva). Rev Fac Nac Salud Pública. 2013;31(supl 1):S13-27.

23. Breilh J. Las tres 'S’ de la determinación de la vida: 10 tesis hacia una visión crítica de la determinación social de la vida y la salud. En: Nogueira RP, organizador. Determinação social da saúde e reforma sanitária. Rio de Janeiro: Cebes; 2010.

24. Israel B, Eng E, Schulz A, Parker E. Methods in community-based participatory research for health. San Francisco: Jossey Bass; 2005.

25. Israel B, Schulz A, Parker E, Becker A. Review of community-based research: assessing partnership approaches to improve public health. Ann Rev Public Health. 1998;19(1):173202.

26. Antunes J, Fernandes da Silva A, Bezerra de Araújo Silva AC, Fernandes de Queiroz Z. Diagnóstico rápido participativo como método de pesquisa em educação. Avaliação, Campinas; Sorocaba. 2018;23(3):590-610. https://doi.org/10.1590/s141440772018000300002

27. Grupo de Trabajo para la Salud y Desarrollo Comunitario de la Universidad de Kansas. Caja de herramientas comunitarias: sesión 3. Llevar a cabo foros públicos y sesiones de escucha [internet]. [Citado 2018 jun 24]. Disponible en: http://ctb.ku.edu/es/tabla-de-contenidos/valoracion/valorar-las-necesidades-y-recursos-comunitarios/foros-de-conducta-publica/principal

28. Wang C, Burris M. Photovoice: concept, methodology, and use for participatoryneeds assessment. Health Educ Behav. 1997;24(3):369-87. https://doi.org/10.1177/109019819702400309

29. Strauss A, Corbin J. Bases de la investigación cualitativa: técnicas y procedimientos para desarrollar la teoría fundamentada. Medellín: Editorial Universidad de Antioquia; 2002.

30. Resolución 8430 de 1993, por la cual se establecen las normas científicas, técnicas y administrativas para la investigación en salud. Diario Oficial 46.506 (4 de octubre de 1993).

31. Asociación Médica Mundial. Declaración de Helsinki: principios éticos para las investigaciones médicas en seres humanos; 2008.

32. Consejo de Organizaciones Internacionales de las Ciencias Médicas. Pautas éticas internacionales para la investigación biomédica en seres humanos. Ginebra; 2002.

33. Emanuel E. ¿Qué hace que la investigación clínica sea ética?: siete requisitos éticos. En: Pellegrino Filho A, Macklin R, editores. Investigación en sujetos humanos: experiencia internacional. Santiago de Chile: Programa Regional de Bioética ops/oms; 1999.

34. Australian Institute of Aboriginal and Torres Strait Islander Studies. Guidelines for ethical research in Australian indigenous studies. 2. ${ }^{a}$ ed. Sídney: Aiatsis; 2012.

35. Breilh J, Tillería Y. Aceleración global y despojo en Ecuador: el retroceso del derecho a la salud en la era neoliberal. Quito: Abya-Yala; 2009.

36. Parra AM. La Guajira: ¿ausencia de Estado? Palabras al Margen [internet]. 2016 ene 14 [citado 2019 ene 02]. Disponible en: http://palabrasalmargen.com/index.php/articulos/ nacional/item/la-guajira-ausencia-de-estado-copy 
37. Lander E. ¿Un nuevo periodo histórico?: crisis civilizatoria, límites del planeta, desigualdad, asaltos a la democracia, estado de guerra permanente y pueblos en resistencia. Foro Social Temático Porto Alegre; enero 2012.

38. Touraine A. Las luces de la razón: la ideología occidental. En: Crítica de la modernidad. Buenos Aires: Fondo de Cultura Económica; 1994. p. 17-38.

39. Orduz N. Desviación del río Ranchería: ¿sí o no? La Silla Vacía [internet]. 2012 ago 12 [citado 2016 dic 20]. Disponible en: https://lasillavacia.com/historia/desviacion-del-riorancheria-si-o-no-35572

40. Rodríguez Rodríguez F. La desviación río Ranchería: ¿un crimen de lesa humanidad? Extractivismo en Colombia [internet]. 2015 oct 8 [citado 2017 ene 2]. Disponible en: http://extractivismoencolombia.org/la-desviacion-del-rio-rancheria-un-crimen-de-lesa-humanidad/

41. Restrepo BN, Restrepo MT, Beltrán JC, Rodríguez M, Ramírez RE. Estado nutricional de niños y niñas indígenas de hasta seis años de edad en el resguardo embera-katío, Tierralta, Córdoba, Colombia. Biomédica. 2006;26(4):517-27. https://doi.org/10.7705/ biomedica.v26i4.317

42. Quintero A. El Quimbo: más allá de la hidroeléctrica: proyecto curricular ingeniería forestal. Boletín Semillas Ambientales. 2016;10(1):66-8.

43. Colombia, Gobernación de La Guajira. Plan de Desarrollo 2016-2019-Gobernación de La Guajira. “Oportunidad para todos y propósito de país”. Riohacha; 2016.

44. Declaración de Atitlán, Guatemala. Consulta de los pueblos indígenas sobre el derecho a la alimentación: una consulta global [internet]. 2002 abr 17-19 [citado 2018 dic 20]. Disponible en: http://www.oda-alc.org/documentos/1341802940.pdf

45. The Lancet. Maternal and child nutrition: executive summary [internet]. 2013 jun 6 [citado 2018 ene 02]. Disponible en: http://www.thelancet.com/series/maternal-and-child-nutrition

46. Organización Mundial de la Salud. ¿Hasta qué edad es adecuado alimentar al bebé solo con leche materna? [Internet]. [Consultado 2019 ene 02]. Disponible en: http://www. who.int/features/qa/21/es/

47. Niño L. Caracterización de la lactancia materna y factores asociados en Puerto Carreño, Colombia. Rev Salud Pública. 2014;16(4):560-71.https://doi.org/10.15446/rsap.v16n4.40653

48. Mintz S. Sabor a comida, sabor a libertad: inclusiones en la comunidad, la cultura y el pasado. Ciudad de México: Ediciones de la Reina Roja; 2003.

49. Rivera J. La satisfacción colectiva de las necesidades de alimentación-nutrición y su relación con la salud-enfermedad. En: Jarillo E, Guinsberg E. La salud colectiva en México: temas y desafíos. Buenos Aires: Lugar Editorial; 2007. p. 153-69.

50. Morales J. El hambre al servicio del neoliberalismo. Bogotá: Desde Abajo; 2006. 